\title{
Sensitization potential and potency of terpene hydroperoxides in the COCAT method
}

Jennifer $\underline{\text { Hennen }}^{1}$, Marta Silva e Sousa ${ }^{1}$, Fatma $_{\text {Sahli }^{2}}$, Jutta Lichter $^{1}$, Jean-Pierre Lepoittevin ${ }^{2}$, Elena

Giménez-Arnau $^{2}$, Brunhilde Blömeke $^{1}$

${ }^{1}$ Department of Environmental Toxicology, Trier University, Trier, Germany.

${ }^{2}$ Dermatochemistry Laboratory, Institute of Chemistry - CNRS UMR 7177, University of Strasbourg, France

Corresponding author: Prof. Dr. Brunhilde Blömeke (PhD)

Department of Environmental Toxicology, Trier University

Universitätsring 15, 54296 Trier, Germany

Phone: 0049-651-201-3782, Fax: 0049-651-201-3780,Email: bloemeke@uni-trier.de

\section{Disclosures:}

\section{Funding:}

The project (DEFCHEMSKALL) is supported by the Deutsche Forschungsgemeinschaft (DFG 284100702, BL360-6) and the Agence Nationale de la Recherche (ANR-15-CE15-0023-01).

\section{Conflict of interest:}

The authors declare that there are no conflicts of interest.

\section{Author contributions:}

$\mathrm{JH}, \mathrm{JL}$ and $\mathrm{BB}$ designed the experiments. MSS and $\mathrm{JH}$ performed the experiments. FS and EGA synthesized the hydroperoxides. All authors contributed significantly to the interpretation of data and writing of the manuscript. All authors consent to the final, submitted manuscript.

Running head: Sensitizing potency of hydroperoxides 


\begin{abstract}
Background: Positive patch tests to mixtures of oxidized terpenes containing allergenic hydroperoxides are frequently reported. However, human sensitization data for these hydroperoxides are not available.
\end{abstract}

Objectives: To analyze and evaluate human sensitization potential and potency of hydroperoxides in vitro using human cells.

Materials/Methods: Limonene-1-hydroperoxide, limonene-2-hydroperoxide, citronellol-7hydroperoxide, cumene hydroperoxide, 1-(1-hydroperoxy-1-methylethyl)cyclohexene and mixtures of citronellol hydroperoxides (isomers at positions 6/7) and linalool hydroperoxides (isomers at positions 6/7) were studied. All compounds were synthesized with the exception of cumene hydroperoxide that was commercial. Their potential and potency to activate dendritic cells (DC) was evaluated by measuring the upregulation of CD86 and CD54 on THP-1 cells upon exposure in the cocultured activation test (COCAT) consisting of HaCaT (human keratinocyte cell line) and THP-1 monocytes (as surrogate for DC).

Results: Hydroperoxides upregulated CD86 and/or CD54 on cocultured THP-1 cells in a concentration-dependent manner. Obtained results are comparable with their sensitization potency ranking in predictive animal models.

Conclusions: For the first time, human sensitization potential and potency of several hydroperoxides were determined using human cells by the COCAT method.

Key words: in vitro, human skin sensitization potential, sensitization potency, fragrances, hydroperoxides, Cocultured Activation Test, COCAT 


\section{Introduction}

Allergic contact dermatitis is the clinical expression of contact allergy, an altered immune status of an individual induced by a particular sensitizing substance, a contact allergen. Upon uptake into the living epidermis, a contact allergen needs to provide an antigen and strongly activate the innate immune system of the skin, providing inflammatory and danger signals from keratinocytes (1-3). These signals allow dendritic cells to leave the skin and mature in the course of their migration to skin-draining lymph nodes where they activate specific naïve $\mathrm{T}$ cells, resulting in expansion of allergen-responsive $\mathrm{T}$ cells. At this point, an individual is immunologically sensitized. Only upon re-exposure with the same, or a cross-reacting, contact allergen, the elicitation phase is triggered, leading to specific $\mathrm{T}$ cell activation with clinical symptoms.

Fragrance compounds remain the most commonly detected allergens in consecutive dermatitis patients, although they are typically moderate sensitizers (4). The prevalence rates of contact allergy to fragrances in women are about twice of those in men (5). Specifically terpene fragrances are ingredients in up to $60 \%$ of cosmetic and household products, and occur naturally in essential oils. Contact allergy to oxidation products of fragrance terpenes such as linalool and limonene are frequently recorded (6-11), while patch test studies for air-oxidized citronellol are absent. Linalool, limonene and citronellol are not contact allergens based on human and animal data $(12,13)$. The sensitizing potency on the basis of the murine local lymph node assay (LLNA) is categorized into extreme (EC3 value of $\leq 0.2 \%)$, strong $(>0.2 \%$ to $\leq 2 \%)$, and moderate $(>2 \%)$. The allylic hydroperoxides derived from air-oxidized linalool (15), limonene (16), citronellol (17) as well as the other air-oxidized compounds were evaluated in the LLNA and results showed that the hydroperoxides are the most potent sensitizers among all oxidation products derived from airoxidation of the parent terpenes. Consequently, limonene-1-hydroperoxide, limonene-2-hydroperoxide (16) and the mixture of linalool-hydroperoxides (15) are categorized as strong sensitizers, and the mixture of citronellol hydroperoxides as moderate (17) (see Tab. 2). Furthermore, exposure to hydroperoxides may also occur due to their use as synthetic chemicals. For instance, cumene hydroperoxide is used in the production of acetone and phenol and as polymerization initiator. 
Overall, the most frequently studied fragrance hydroperoxides are limonene-2-hydroperoxide (lim-2$\mathrm{OOH}$ ), linalool-6-hydroperoxide (lin-6-OOH) and linalool-7-hydroperoxide (lin-7-OOH). The quantification of hydroperoxides in consumer products is challenging due to low thermal stability and their lack of chromophores (18-21). The link between exposure to the hydroperoxides and allergic contact dermatitis is well documented only in few cases. For example, a 7-year-old girl developed severe eyelid dermatitis after using a shampoo, which contained linalool and low levels $(0.2 \mu \mathrm{g} / \mathrm{g})$ of lin-OOH (22).

The aim of the present study was to investigate the sensitizing potency of a series of hydroperoxides by using our Cocultured Activation Test (COCAT), developed to identify the human skin sensitization hazard and determination of skin sensitization potency in vitro $(23,24)$. COCAT is based on the use of cocultures of HaCaT (human keratinocyte cell line) and THP-1 cells (surrogate of antigen presenting cells). Individually, these cell lines are used to address key steps in the process of sensitization, namely activation of keratinocytes (key event 2 ) and activation and maturation of dendritic cells (key event 3) of the skin sensitization Adverse Outcome Pathway. We found that exposure on the cocultured cells has the potential to increase their response to sensitizing chemicals, enabling the detection of prohaptens and supporting the identification of skin sensitization potency $(24,26,27)$. Additionally, a 79.5\% (31/39) concordance was found when estimating potency based on a prediction of 4 potency subcategories (extreme/strong, moderate, weak, non-sensitizer) by linear interpolation of the lowest concentration needed to reach positivity for estimated markers (CD54 or CD86) as compared to LLNA EC3 values (28). 


\section{Materials and Methods}

\section{Chemicals}

The following compounds were studied: limonene-1-hydroperoxide [(1S, 4R)-4-isopropenyl-1methyl-2-cyclohexene-1-hydroperoxide (lim-1-OOH)], limonene-2-hydroperoxide [(5R)-5isopropenyl-2-methyl-2-cyclohexene-1-hydroperoxide (lim-2-OOH)], citronellol-7-hydroperoxide [(E)-7-hydroperoxy-3,7-dimethyloct-5-ene-1-ol (citr-7-OOH)], mixture of citronellol hydroperoxides [6-hydroperoxy-3,7-dimethyloct-7-ene-1-ol (citr-6-OOH)] and citr-7-OOH (citr-6/7-OOH, ratio 6:4), mixture of linalool hydroperoxides [6-hydroperoxy-3,7-dimethylocta-1,7-diene-3-ol (lin-6-OOH)] and [7-hydroperoxy-3,7-dimethylocta-1,5-diene-3-ol (lin-7-OOH)] in a 6:4 ratio (lin-6/7-OOH), cumene hydroperoxide (cum-OOH, Sigma-Aldrich, Saint Quentin Fallavier, France), 1-(1-hydroperoxy-1methylethyl)cyclohexene (cyc-hex-OOH), sodium dodecyl sulfate (SDS, Carl Roth, Karlsruhe, Germany) and 2,4-dinitrochlorobenzene (DNCB, Sigma Aldrich Taufkirchen, Germany).

The synthesis of the hydroperoxides was performed in the Dermatochemistry Laboratory (Strasbourg, France). Lim-1-OOH and lim-2-OOH hydroperoxides were synthesized as reported previously in the literature $(29,30)$. For the mixture citr-6/7-OOH we have followed exactly the procedure we described for lin-6/7-OOH and obtained for both mixtures an isomer ratio of 6:4 (30). Cyc-hex-OOH was synthesized following also a method described in the literature (31). All synthesized compounds were pure according to the ${ }^{1} \mathrm{H}$ and ${ }^{13} \mathrm{C}$ NMR characterizations, in agreement with the literature mentioned. Chemical structures of studied hydroperoxides are given in Fig. 1.

\section{Cocultured activation test (COCAT)}

THP-1 cells $\left(8 \times 10^{4}, 96\right.$-well $)$ were placed on top of confluent HaCaT keratinocytes. The maximal test concentration (up to $4000 \mu \mathrm{M}$ ) for each test chemical is determined based on solubility in the culture medium and/or cell viability. Cocultured cells were exposed to increasing concentrations of the compounds $(7.8-4000 \mu \mathrm{M})$ dissolved in final concentration of $0.2 \% \mathrm{DMSO}$. Afterwards cell viability was determined and concentrations inducing not more than 30-35\% reduction of THP-1 cell viability 
were used for the experiments. Overall 7-9 concentrations were evaluated for each hydroperoxide (see Tab. 1). In each run, $20 \mu \mathrm{M}$ of 2,4-dinitrochlorobenzene (DNCB) and $144 \mu \mathrm{M}$ sodium dodecyl sulfate (SDS) were used as positive and negative control, respectively. After $24 \mathrm{~h}$ of treatment, floating THP1 cells were harvested and cell surface expression (mean fluorescence intensities, MFI) of CD86 and CD54 was analyzed on viable THP-1 cells (DAPI/propidium iodide negative) via flow cytometry (FACSVerse $^{\mathrm{TM}}$ or FACSCalibur ${ }^{\mathrm{TM}}$, BD Biosciences, Heidelberg, Germany). Briefly, THP-1 cells were stained with fluorescein isothiocyanate (FITC)-labeled anti-CD86 (clone 2331 [FUN-1]), APC-labeled anti-CD54 (clone HA58) monoclonal antibodies (all mouse IgG1, obtained from BD Pharmingen, Heidelberg, Germany) or corresponding isotype controls. Then, the chemical-induced differences in cell surface levels of CD86 or CD54 ( $\triangle \mathrm{MFI}$ ) on viable THP-1 (determined by DAPI/propidium iodide exclusion) were calculated for each sample. A $\triangle$ MFI of 10.8 for CD86 and 300 for CD54 were used as thresholds to identify a sensitizer. These thresholds were established by analyzing a set of 21 sensitizers and 12 non-sensitizers in the course of the method development. The lowest EC $\Delta$ (either CD54 or CD86) for each run was used to estimate potency. Mean EC $\Delta$ and standard error were calculated for each compound $(24,28)$. 


\section{Results}

We investigated the human sensitization potential and potency using the COCAT method. Specifically, limonene-1-hydroperoxide (lim-1-OOH), limonene-2-hydroperoxide (lim-2-OOH), citronellol-7-hydroperoxide (citr-7-OOH), mixture of citronellol hydroperoxides (citr-6-OOH) and (citr-7-OOH) in a 6:4 ratio (citr-6/7-OOH), mixture of linalool hydroperoxides (lin-6-OOH) and (lin7-OOH) in a 6:4 ratio (lin-6/7-OOH), cumene hydroperoxide (cum-OOH), and 1-(1-hydroperoxy-1methylethyl)cyclohexene (cyc-hex-OOH) were investigated (see Fig. 1). Representative flow cytometry histogram plots are shown in Fig. 2. All studied hydroperoxides upregulated both markers for activation of dendritic cells, CD86 and CD54, on cocultured THP-1 cells in a concentrationdependent manner. The corresponding curves for CD86 and CD54 are shown in Fig. 3 A-G. Furthermore, all compounds reached the threshold for positivity (CD86: $\triangle$ MFI of 10.8, CD54: $\Delta$ MFI of 300) for one or two markers in at least 2 out of 3 runs. All hydroperoxides were correctly predicted as sensitizers. The lowest concentration needed to reach a threshold for positivity (effective concentration, $\mathrm{EC} \Delta$ ) was used to estimate potency of the molecule (24). Here we found that the tested hydroperoxides needed similar concentrations to reach positivity, values varied between 0.29 and 0.65 mM. In contrast, for DNCB classified as an extreme sensitizer in the LLNA, at least a 10-fold lower concentration was sufficient to reach positivity (Tab. 2). In case of the limonene hydroperoxides, we found lim-1-OOH more potent than lim-2-OOH, similarly to the LLNA data. The values for the lin6/7-OOH are close to lim-2-OOH. Interestingly, citr-7-OOH alone was less potent than the mixture of citr-6/7-OOH (ratio 6:4). The concentrations needed for positivity of cum-OOH and cyc-hex-OOH were comparable to values found for lim-2-OOH (Tab. 3). 


\section{Discussion}

In this study, we analyzed the skin sensitization potential and potency of allergenic hydroperoxides in vitro using our COCAT method $(24,26-28)$. All hydroperoxides were correctly classified as skin sensitizers based on analysis of DC activation in their natural environment of keratinocytes. We found a concentration-dependent upregulation of CD86 and CD54 over a wide range of concentrations. The concentration needed for positivity ranged between 0.29 and $0.65 \mathrm{mM}$. Thus, our potency ranking is comparable to reported in vivo data from predictive animal testing (Tab. 2 and 3). The highest potency was found for lim-1-OOH followed by lim-2-OOH. Although exploration of the mechanism(s) leading to the upregulation of the two markers CD86 and CD54 was not the focus of this study, it seems reasonable to speculate that the difference might reflect the different capacity of the two molecules to generate radicals. For instance, a higher ability of lim-1-OOH to generate different kinds of radicals was detected using radical trapping experiments (32). For lim-1-OOH both carbon-centered and oxygen-centered radicals were trapped, while experiments with lim-2-OOH retrieved only small amounts of carbon-centered radicals. Instead, the main product trapped in experiments testing lim-2$\mathrm{OOH}$ was carvone. But carvone is much less sensitizing in animal experiments (LLNA EC3 value $0.86 \mathrm{M}, 13 \% \mathrm{w} / \mathrm{v}(33)$, and cannot account for the results obtained with COCAT. This notion is also supported by the EC $\Delta$ value of carvone $(0.55 \pm 0.11 \mathrm{mM})$ in COCAT (28). In addition, published prevalence of positive patch test reactions to oxidized fragrance hydroperoxides (34) is in accordance with the potency ranking obtained in this study. Specifically, a human patch test study including 763 eczema patients all tested with lim-1-OOH and lim-2-OOH showed that 18 individuals responded to lim-1-OOH and 13 individuals to lim-2-OOH (34).

Our results for lim- $\mathrm{OOH}$ and lin- $\mathrm{OOH}$ are in general agreement with a previous study investigating THP-1 activation in the absence of keratinocytes among further in-depth mechanistically aspects (35). Looking at the correlation of the compound concentrations, we needed much lower concentrations in the presence of keratinocytes. This suggests that keratinocytes support THP-1 activation. A different responsiveness between mono- and coculture of THP-1 cells was already observed with a different set 
of chemicals $(24,28)$ where compared to exposing THP-1 alone, coculturing resulted in up to 3 -fold enhanced maximal CD86 and/or CD54 upregulation on THP-1.

$\underline{\text { Cum-OOH and cyc-hex-OOH are quite comparably potent in COCAT. The comparable potency of }}$ cum-OOH and cyc-hex-OOH with lim-2-OOH observed in COCAT is in good agreement with in vivo results also indicating comparable potencies of these molecules (31). The lowest potency was detected for citr-7-OOH. Citronellol belongs also to the highly used fragrances in terms of the relative volume sold (36). With respect to its oxidation products it was demonstrated that citr-7-OOH together with citr-6-OOH contributes the most to the sensitizing potency of autoxidized citronellol in the LLNA (17). Comparison of our results for citr-7-OOH alone with the results gained for citr-6/7-OOH indicate that citr-7-OOH may have influenced the results obtained for the mixture. This is in contrast to results reported for lin-6/7-OOH and lin-7-OOH exposing THP-1 alone, where no difference was found for $\underline{\text { lin-7-OOH and the lin-6/7-OOH (35). Besides the different experimental setups, there is no obvious }}$ difference in the structure of citr-7-OOH and lin-7-OOH but clearly more detailed structure-related analysis are necessary to explain the observed differences depending on chemical structures.

At present, comparison of our data for the hydroperoxides with results from other alternative methods explicitly evaluating the potency of these compounds is not possible due to a lack of data. In addition no meaningful results were received from an in silico tool available (DC ITS at https://its.douglasconnect.com).

In sum, this study is the first evaluating the human sensitization potential and potency of several hydroperoxides, mainly fragrance hydroperoxides, in vitro with the COCAT method. The results obtained are in line with the results from the literature using in vivo methods. These data, together with our earlier results $(23,24,26-28)$ demonstrate that COCAT can support the assessment of chemicals with as yet limited or absent human sensitization data. 
New Tab. 1: Concentrations of hydroperoxides used for the experiments.

\begin{tabular}{|l|c|c|c|}
\hline Compound & $\begin{array}{c}\text { Solvent } \\
(0.2 \% \text { final } \\
\text { conc. })\end{array}$ & $\begin{array}{c}\text { Max. concentration }(\mu \mathrm{M}) \text { resulting } \\
\text { in 30-35\% reduction of THP-1 cell } \\
\text { viability }\end{array}$ & $\begin{array}{c}\text { Total number of } \\
\text { tested } \\
\text { concentrations }\end{array}$ \\
\hline Lim-1-OOH & DMSO & 500 & 9 \\
\hline Lim-2-OOH & DMSO & 500 & 7 \\
\hline Lin-6/7-OOH & DMSO & 500 & 8 \\
\hline Citr-6/7-OOH & DMSO & 1000 & 9 \\
\hline Citr-7-OOH & DMSO & 1000 & 9 \\
\hline Cyc-hex-OOH & DMSO & 1000 & 9 \\
\hline Cum-OOH & DMSO & 500 & \\
\hline
\end{tabular}


Tab. 2: Comparison of the skin sensitization potencies of fragrance terpene hydroperoxides and 2,4-dinitrochlorobenzene (DNCB) in vitro (COCAT) and in vivo (LLNA).

\begin{tabular}{|l|c|c|c|}
\hline Compound & $\begin{array}{c}\text { COCAT EC } \Delta \\
(\mathrm{mM}, \text { mean } \pm \mathrm{SEM})^{\dagger}\end{array}$ & $\begin{array}{c}\text { Human sensitization } \\
\text { category }\end{array}$ & $\begin{array}{c}\text { LLNA EC3 } \\
(\mathrm{mM} ; \% \mathrm{w} / \mathrm{v})\end{array}$ \\
\hline Lim-1-OOH & $0.29 \pm 0.01$ & n.a. & $19 ; 0.33^{1}$ \\
\hline Lim-2-OOH & $0.35 \pm 0.08$ & n.a. & $49 ; 0.83^{1}$ \\
\hline Lin-6/7-OOH, ratio 3:5 & $\underline{\text { n.a. }}$ & $\underline{\text { n.a. }}$ & $\underline{86 ; 1.6^{2}}$ \\
\hline Lin-6/7-OOH, ratio 6:4 & $0.36 \pm 0.04$ & n.a. & n.a. \\
\hline Citr-6/7-OOH, ratio 6:4 & $0.29 \pm 0.07$ & n.a. & $100 ; 2.3^{3}$ \\
\hline Citr-7-OOH & $0.65 \pm 0.17$ & n.a. & n.a. \\
\hline DNCB & $0.014 \pm 0.0037$ & 1 & $2.96 ; 0.06^{4}$ \\
\hline
\end{tabular}

$n=3$, except $n=2$ for citr-6/7-OOH. n.a., not available

${ }^{1}$ Ref. (16)

${ }^{2}$ Ref. (15)

${ }^{3}$ Ref. (17)

${ }^{4}$ Ref. (37) 
Tab. 3: Comparison of the skin sensitization potencies of lim-2-OOH, cum-OOH and cyc-hex-OOH in vitro (COCAT) with published in vivo results derived from guinea pig test (Freund's complete adjuvant test, FCAT).

\begin{tabular}{|l|c|c|c|}
\hline Compound & COCAT & \multicolumn{2}{|c|}{ guinea pig test (FCAT) } \\
\hline & EC $\Delta$ & $\underline{\text { Sensitized animals }^{1}}$ & $\underline{\text { Sensitized animals }^{1}}$ \\
& $(\mathrm{mM}, \text { mean } \pm \mathrm{SEM})^{\dagger}$ & $\begin{array}{c}\%) \text { after challenge } \\
\text { with } 0.3 \%\end{array}$ & $\begin{array}{c}\text { (\%) after challenge } \\
\text { with } 1 \%\end{array}$ \\
\hline$\underline{\text { Lim-2-OOH }}$ & $0.35 \pm 0.08$ & $\underline{33.3^{2}}$ & $\underline{100^{2}}$ \\
\hline$\underline{\text { Cum-OOH }}$ & $0.30 \pm 0.12$ & $\underline{13.3^{3}}$ & $\underline{86.7^{3}}$ \\
\hline$\underline{\text { Cyc-hex-OOH }}$ & $0.38 \pm 0.01$ & $\underline{25^{4}}$ & $\underline{91.7^{4}}$ \\
\hline
\end{tabular}

$n=3$, except $n=2$ for cum-OOH

${ }^{1}$ at $72 \mathrm{~h}$ after challenge

${ }^{2}$ induction was performed with $1.5 \% \mathrm{lim}-2-\mathrm{OOH}(38)$

${ }^{3}$ induction was performed with $1 \%$ cum-OOH (38)

${ }^{4}$ induction was performed with $3 \%$ cyc-hex-OOH (31) 


\section{Figure legends}

Fig. 1 Chemical structures of terpene hydroperoxides included in this study: lim-1-OOH (1), lim2-OOH (2), lin-6-OOH (3), lin-7-OOH (4), citr-6-OOH (5), citr-7-OOH (6), cum-OOH (7), cyc-hexOOH (8).

New Fig. 2 Representative flow cytometry histograms for the geometric mean fluorescence intensity (MFI) of CD86 and CD54. Expression of CD86 and CD54 on cocultured THP-1 cells after treatment with solvent ( $0.2 \%$ DMSO, A), $500 \mu \mathrm{M}$ lim-1-OOH (B), $750 \mu \mathrm{M}$ citr-6/7-OOH (C) and 20 $\mu \mathrm{M}$ DNCB (D). Flow cytometry histograms show the number of events versus FITC (CD86) and APC (CD54) fluorescence intensities. Solid lines represent histograms for the indicated antibodies (FITClabeled anti-CD86 or APC-labeled anti-CD54), dotted lines represent histograms of the corresponding isotype controls (FITC or APC labeled IgG1), respectively.

Fig. 3 Concentration-dependent upregulation of CD86 and CD54 by hydroperoxides. Cocultured HaCaT and THP-1 cells were exposed to limonene-1-hydroperoxide (lim-1-OOH, A), limonene-2hydroperoxide (lim-2-OOH, B), a mixture of linalool-6/7-hydroperoxides (lin-6/7-OOH, C), a mixture of citronellol-6/7-hydroperoxides (citr-6/7-OOH, D), citronellol-7-hydroperoxide (citr-7-OOH, E), cumene hydroperoxide (cum-OOH, F) and cyclohexene-hydroperoxide (cyc-hex-OOH, G) for $24 \mathrm{~h}$. Afterwards, the expression of CD86 and CD54 was analyzed on harvested THP-1 cells via flow cytometry and given as chemical-induced differences in cell surface levels of CD86 or CD54 (mean \pm SEM of $n=3$; citr-6/7-OOH and cum-OOH, $n=2$ ). 
References (neue und verbesserte Ref. unterstreichen nicht vergessen)

1 Banchereau J, Steinman R M. Dendritic cells and the control of immunity. Nature. $1998 ; 392: 245-52$.

2 Iwasaki A, Medzhitov R. Toll-like receptor control of the adaptive immune responses. Nat Immunol. 2004;5:987-95.

3 Banchereau J, Thompson-Snipes L, Zurawski S et al. The differential production of cytokines by human Langerhans cells and dermal CD14(+) DCs controls CTL priming. Blood. 2012;119:5742-9.

4 Liden C, Yazar K, Johansen J D, Karlberg A T, Uter W, White I R. Comparative sensitizing potencies of fragrances, preservatives, and hair dyes. Contact Dermatitis. 2016;75:265-275.

5 Diepgen $\mathrm{T}$ L, Ofenloch R, Bruze $\mathrm{M}$ et al. Prevalence of fragrance contact allergy in the general population of five European countries: a cross-sectional study. $\mathrm{Br} J$ Dermatol. 2015;173:1411-9.

6 Deza G, Garcia-Bravo B, Silvestre J F et al. Contact sensitization to limonene and linalool hydroperoxides in Spain: a GEIDAC* prospective study. Contact Dermatitis. 2017;76:74-80.

7 Wlodek C, Penfold C M, Bourke J F et al. Recommendation to test limonene hydroperoxides $0.3 \%$ and linalool hydroperoxides $1.0 \%$ in the British Baseline patch test series. $\mathrm{Br} J$ Dermatol. 2017;177:1708-1715.

8 Nath N S, Liu B, Green C, Atwater A R. Contact Allergy to Hydroperoxides of Linalool and D-Limonene in a US Population. Dermatitis. 2017;28:313-316.

9 Audrain H, Kenward C, Lovell C R et al. Allergy to oxidized limonene and linalool is frequent in the U.K. Br J Dermatol. 2014;171:292-297.

10 Bråred Christensson J, Andersen K E, Bruze M et al. Air-oxidized linalool: a frequent cause of fragrance contact allergy. Contact Dermatitis. 2012;67:247-59.

11 Bråred Christensson J, Andersen K E, Bruze M et al. An international multicentre study on the allergenic activity of air-oxidized R-limonene. Contact Dermatitis. 2013;68:214-23.

12 Kern P, Gerberick G, Ryan C, Kimber I, Aptula A, Basketter D. Local lymph node data for the evaluation of skin sensitization alternatives: a second compilation. Dermatitis. 2010;21:8-32.

13 Api A M, Basketter D, Lalko J. Correlation between experimental human and murine skin sensitization induction thresholds. Cutan Ocul Toxicol. 2015;34:298-302.

14 European Commission, Scientific Committee on Consumer Products. Memorandum on classification and categorization of skin sensitisers and grading of test reactions, 2005. https://ec.europa.eu/health/ph_risk/committees/04_sccp/docs/sccp_s_01.pdf. Accessed February 28, 2019. 

linalool. Identification and quantification of primary and secondary oxidation products and the effect on skin sensitization. Chem Res Toxicol. 2004;17:1697-1705. Johansson S, Gimenez-Arnau E, Grotli M, Karlberg A T, Borje A. Carbon- and oxygencentered radicals are equally important haptens of allylic hydroperoxides in allergic contact dermatitis. Chem Res Toxicol. 2008;21:1536-47.

17 Rudbäck J, Hagvall L, Börje A, Nilsson U, Karlberg A T. Characterization of skin sensitizers from autoxidized citronellol - impact of the terpene structure on the autoxidation process. Contact Dermatitis. 2014;70:329-39.

18 Kern S, Dkhil H, Hendarsa P, Ellis G, Natsch A. Detection of potentially skin sensitizing hydroperoxides of linalool in fragranced products. Anal Bioanal Chem. 2014;406:6165-78. Kern S, Granier T, Dkhil H, Haupt T, Ellis G, Natsch A. Stability of limonene and monitoring of a hydroperoxide in fragranced products. Flavour Fragr J. 2014;29:277-286.

20 Rudbäck J, Islam M N, Börje A, Nilsson U, Karlberg A T. Essential oils can contain allergenic hydroperoxides at eliciting levels, regardless of handling and storage. Contact Dermatitis. 2015;73:253-4.

21 Ramzi A, Ahmadi H, Sadiktsis I, Nilsson U. A two-dimensional non-comprehensive reversed/normal phase high-performance liquid chromatography/tandem mass spectrometry system for determination of limonene and linalool hydroperoxides. $J$ Chromatogr A. 2018;1566:102-110.

22 Elliott J F, Ramzy A, Nilsson U, Moffat W, Suzuki K. Severe intractable eyelid dermatitis probably caused by exposure to hydroperoxides of linalool in a heavily fragranced shampoo. Contact Dermatitis. 2017;76:114-115.

23 Hennen J, Aeby P, Goebel C et al. Cross talk between keratinocytes and dendritic cells: impact on the prediction of sensitization. Toxicol Sci. 2011;123:501-10.

24 Hennen J, Blömeke B. Keratinocytes improve prediction of sensitization potential and potency of chemicals with THP-1 cells. ALTEX. 2017;34:279-288.

25 OECD. The Adverse Outcome Pathway for Skin Sensitisation Initiated by Covalent Binding to Proteins, 2014. https://doi.org/10.1787/9789264221444-en. Accessed February 28, 2019.

26 Hennen J, Blömeke B. Assessment of skin sensitization potency of hair dye molecules in vitro. Contact Dermatitis. 2017;77:179-180.

27 Hennen J, Blömeke B. Ranking skin-sensitizing hair dye molecules according to their potency by the use of human cells. Contact Dermatitis. 2018;79:391-393.

28 Hennen J, Lichter J, Schellenberger M et al. Cocultured activation test (COCAT)-Advanced safety assessment of chemical sensitizers. Naunyn Schmiedebergs Arch Pharmacol. 2018;391:S83. 
29 Calandra M J, Impellizzeri J, Wang Y. An HPLC method for hydroperoxides derived from limonene and linalool in citrus oils, using post-column luminol-mediated chemiluminescence detection. Flavour Fragr J. 2015;30:121-130.

30 Kao D, Chaintreau A, Lepoittevin J P, Gimenez-Arnau E. Synthesis of allylic hydroperoxides and EPR spin-trapping studies on the formation of radicals in iron systems as potential initiators of the sensitizing pathway. J Org Chem. 2011;76:6188-200.

31 Lepoittevin J P, Karlberg A T. Interactions of Allergenic Hydroperoxides with Proteins - a Radical Mechanism. Chem Res Toxicol. 1994;7:130-133.

32 Bråred Christensson J, Johansson S, Hagvall L, Jonsson C, Borje A, Karlberg A T. Limonene hydroperoxide analogues differ in allergenic activity. Contact Dermatitis. 2008;59:344-52.

33 Nilsson A M, Bergström M A, Luthman K, Nilsson J L, Karlberg A T. An alpha,betaunsaturated oxime identified as a strong contact allergen. Indications of antigen formation via several pathways. Food Chem Toxicol. 2005;43:1627-36.

34 Bråred Christensson J, Hellsen S, Borje A, Karlberg A T. Limonene hydroperoxide analogues show specific patch test reactions. Contact Dermatitis. 2014;70:291-9.

35 Raffalli C, Clouet E, Kuresepi S et al. Editor's Highlight: Fragrance Allergens Linalool and Limonene Allylic Hydroperoxides in Skin Allergy: Mechanisms of Action Focusing on Transcription Factor Nrf2. Toxicol Sci. 2018;161:139-148.

36 Schnuch A, Uter W, Lessmann H, Geier J. Risk of sensitization to fragrances estimated on the basis of patch test data and exposure, according to volume used and a sample of 5451 cosmetic products. Flavour and fragrance journal. 2015;30:208-217.

37 Hoffmann $\mathrm{S}$, Kleinstreuer $\mathrm{N}$, Alepee $\mathrm{N}$ et al. Non-animal methods to predict skin sensitization (I): the Cosmetics Europe database. Crit Rev Toxicol. 2018:1-15.

38 Bråred Christensson J, Matura M, Backtorp C, Borje A, Nilsson J L, Karlberg A T. Hydroperoxides form specific antigens in contact allergy. Contact Dermatitis. 2006;55:230-7. 